This item was submitted to Loughborough's Research Repository by the author.

Items in Figshare are protected by copyright, with all rights reserved, unless otherwise indicated.

\title{
Active flow control of jet mixing using steady and pulsed fluid tabs
}

PLEASE CITE THE PUBLISHED VERSION

PUBLISHER

Professional Engineering Publishing / @ IMECHE

VERSION

VoR (Version of Record)

LICENCE

CC BY-NC-ND 4.0

REPOSITORY RECORD

Behrouzi, P., T. Feng, and James J. McGuirk. 2009. "Active Flow Control of Jet Mixing Using Steady and Pulsed Fluid Tabs”. figshare. https://hdl.handle.net/2134/4774. 
This item was submitted to Loughborough's Institutional Repository (https://dspace.lboro.ac.uk/) by the author and is made available under the following Creative Commons Licence conditions.

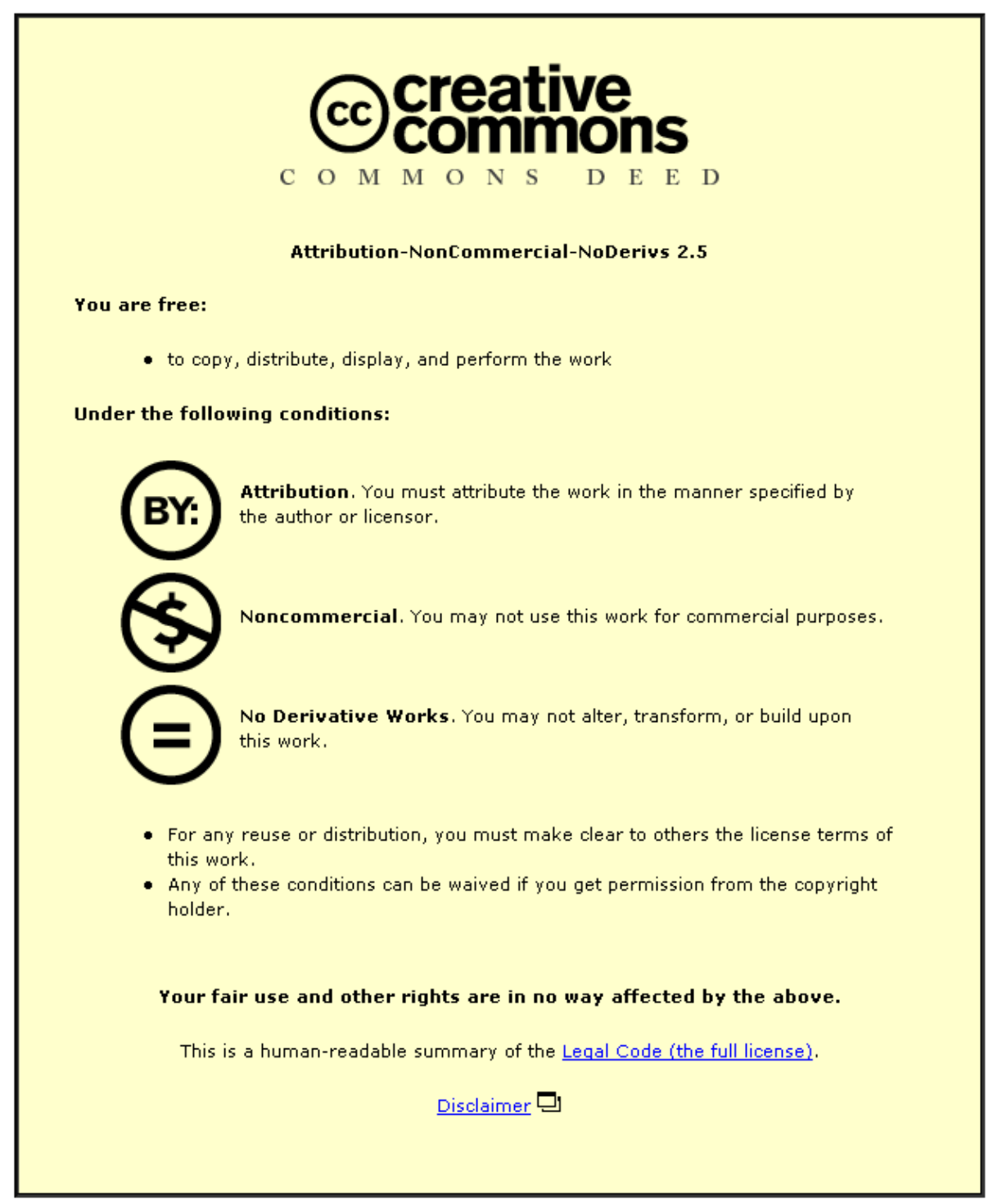

For the full text of this licence, please go to: http://creativecommons.org/licenses/by-nc-nd/2.5/ 


\title{
Active flow control of jet mixing using steady and pulsed fluid tabs
}

\author{
P Behrouzi, T Feng, and J J McGuirk* \\ Department of Aero and Auto Engineering, Loughborough University, Loughborough, UK
}

The manuscript was received on 4 January 2008 and was accepted after revision for publication on 31 March 2008.

DOI: 10.1243/09596518JSCE543

\begin{abstract}
Flow control techniques for increasing the rate of jet mixing in axisymmetric nozzle flows have been investigated. A combination of water tunnel and high-speed airflow facilities is used to assess the near-field jet behaviour. Solid tabs, steady fluid tabs (i.e. discrete radially discharged control jets located close to the core jet exit), and pulsed fluid tabs are compared. The effect of fluid tab velocity amplitude, pulse rate, and pulse phase are studied using openloop control. The measurements indicate that fluid tabs generate a similar streamwise vortex formation process (and hence display increased mixing) as previously observed in solid-tabbed nozzle flows. In incompressible testing the mixing effectiveness with a pair of pulsed fluid tabs $180^{\circ}$ out-of-phase was as good as a twin solid tab nozzle for a control jet flowrate of only 0.5 per cent of the primary (core) jet flow. In preliminary high-speed testing similar benefits of fluid tabs over solid tabs were observed. Further study of pulsed fluid tabs is recommended; they have the attractive performance benefit that they can be easily switched off when not needed and offer increased flexibility as the basis of an optimized active control jet mixing device.
\end{abstract}

Keywords: jet mixing control, steady/pulsed control jets, water tunnel/high-speed air experiments

\section{INTRODUCTION}

A full understanding of the exhaust jet mixing behaviour of aeroengine propulsion nozzles is essential to predict aircraft afterbody aerodynamics. For civil aircraft applications, the engineering design driver is to achieve jet noise reduction. For military engines, there is much interest in the increased drag due to interaction between exhaust jet and aircraft afterbody, and also in jet plume mixing enhancement for infrared signature reduction. Given these motivations, extensive research on jet mixing enhancement and noise suppression has been under way for several decades. Many techniques (both passive and active) have been suggested, such as lobed mixers, solid tabs, acoustic excitation, serrated nozzles, etc. A summary of the major fluid dynamic properties of these devices has been provided by Seiner et al. [1]. In terms of active control, early work

*Corresponding author: Department of Aero and Auto Engineering, Loughborough University, Ashby Road, Loughborough LE11 3TU,UK.email: j.j.mcguirk@lboro.ac.uk was based on the use of acoustic input to excite the jet to mix rapidly. Rockwell [2] studied the active control of low Reynolds number jets in this way and found that when the excitation frequency coincided with the natural breakdown frequency of the jet shear layer, accelerated transition to turbulence and enhanced mixing was observed. Parekh et al. [3] examined several methods of active control (acoustic, fluidic, and mechanical oscillating ribbons) and observed similar effects, including the production of different excited jet modes (flapping, bursting) which produced intense mixing. These attempts at active control have, however, usually been restricted to low Reynolds and Mach number jets. Also, while enhanced mixing was observed, the jet flapping motions were sufficiently large that the impact on thrust loss from the jets would have been unacceptable in the engine exhaust context. Similarly, while acoustic excitation is effective, the power requirements and weight implications to achieve the required effect have so far precluded any significant further interest in this mode of active control for aircraft applications. 
The technique that has therefore received most attention to date is a passive control device involving use of solid tabs [4-7]. Tabs are small protrusions placed at the jet nozzle exit; usually more than one is used, spaced around the jet periphery. Tab-induced flow appears to offer a practical method for mixing enhancement in the jet near field, i.e. in the first 10 jet diameters from the nozzle exit. All the above studies have confirmed the main mechanism by which solid tabs enhance primary (or core) jet mixing as the creation of strong, streamwise vortex motions emanating from the tabs. These distort the jet cross-section, increasing the interfacial area between the jet and the surrounding ambient fluid, and raising the level of turbulent stresses that are responsible for entrainment of ambient mass into the jet. Figure 1 illustrates this mechanism. The streamwise vortices emanating from a nozzle containing two solid tabs are indicated schematically. The induced secondary ( $y-z$ plane) velocities cause ambient fluid to be drawn into the jet along the tab axis ( $y$ direction) and by continuity the jet fluid is pushed into the ambient along the $z$ axis. Both of these processes enhance jet spreading. Evidence of this jet cross-section distortion is provided in Fig. 2. Water tunnel plume visualization data taken from Behrouzi and McGuirk [6] compare an instantaneous jet cross-section for a clean (no tabs) jet with one from a twin-tabbed nozzle; the inwards movement along the (vertical) tab axis and the enhanced jet width along the lateral (horizontal) axis are clear to see. While reference [6] studied only low-speed jets, recent measurements by Feng and McGuirk [8] have provided comprehensive data on the velocity field generated by solid tabs in a high Mach number

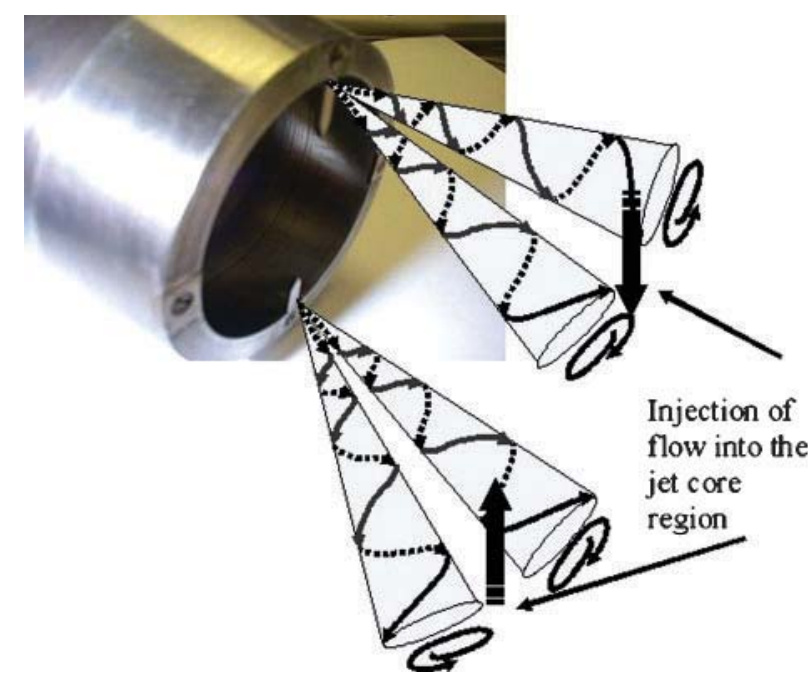

Fig. 1 Streamwise vortices created by a solid tab exhaust plume ( $M=1$, underexpanded jet) as relevant to aeroengines, illustrating clearly the same streamwise vortex formation process, as also shown in Fig. 2 via measured secondary velocity vectors and axial velocity contours (data shown for the upper tab only).

Solid tabs are not the only way to generate streamwise vorticity. Jets in crossflow is probably the classical flow configuration associated with the appearance of streamwise vorticity. There is a large volume of literature on jets in crossflow, since these appear in many engineering applications. Entire conferences have been organized on this subject [9]. One of the most comprehensive summaries of current knowledge is provided by the review of Margason [10] from one conference, covering over 300 papers on the subject. Aspects of relevance to the present application include the derivation of empirical correlations for the jet trajectory, the prime importance of the jet/crossflow momentum flux ratio (or velocity ratio when jet and crossflow fluid have the same density), and the use of nonintrusive laser based methods for quantitative capture of velocity and turbulence information in the downstream jet region. Bray and Garry [11] have reviewed the application of jets in crossflow (referred to by them as air jet vortex generators (AJVGs)) to control flow separation on aerofoils at high angles of attack by encouraging mixing of a high-momentum fluid away from the wall with a low-momentum inner boundary layer fluid. Bons et al. [12] have investigated pulsed AJVGs for use on low-pressure turbine blades and found that pulsing the control jets produced a comparable reduction in boundary layer separation for only a fraction of the mass flow required of steady AJVGs. Behrouzi and McGuirk [13] have applied the concept of steady control jets in crossflow (referred to in their work and in the present paper as 'fluid tabs') to the control of exhaust nozzle jet mixing. Evidence from incompressible experiments and CFD predictions confirmed that steady fluid tabs reproduced similar benefits to solid tabs in terms of jet potential core length reduction and increased centre-line velocity decay rate (these parameters are defined below). This work underlined one additional advantage of fluid tabs. Not only can they be easily switched off if not wanted, but their strength can be adapted to suit different conditions (via their momentum (velocity) ratio). In broad terms observations in compressible and hot jet experiments carried out by Behrouzi and McGuirk [13] confirmed that fluid tab jets behaved in a similar manner for both low-speed and high- 

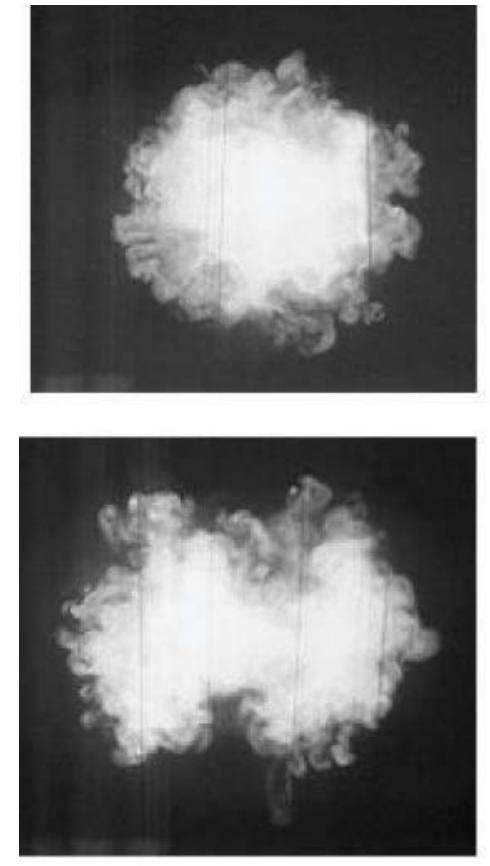

$\mathrm{y} / \mathrm{D}_{\mathrm{n}}$

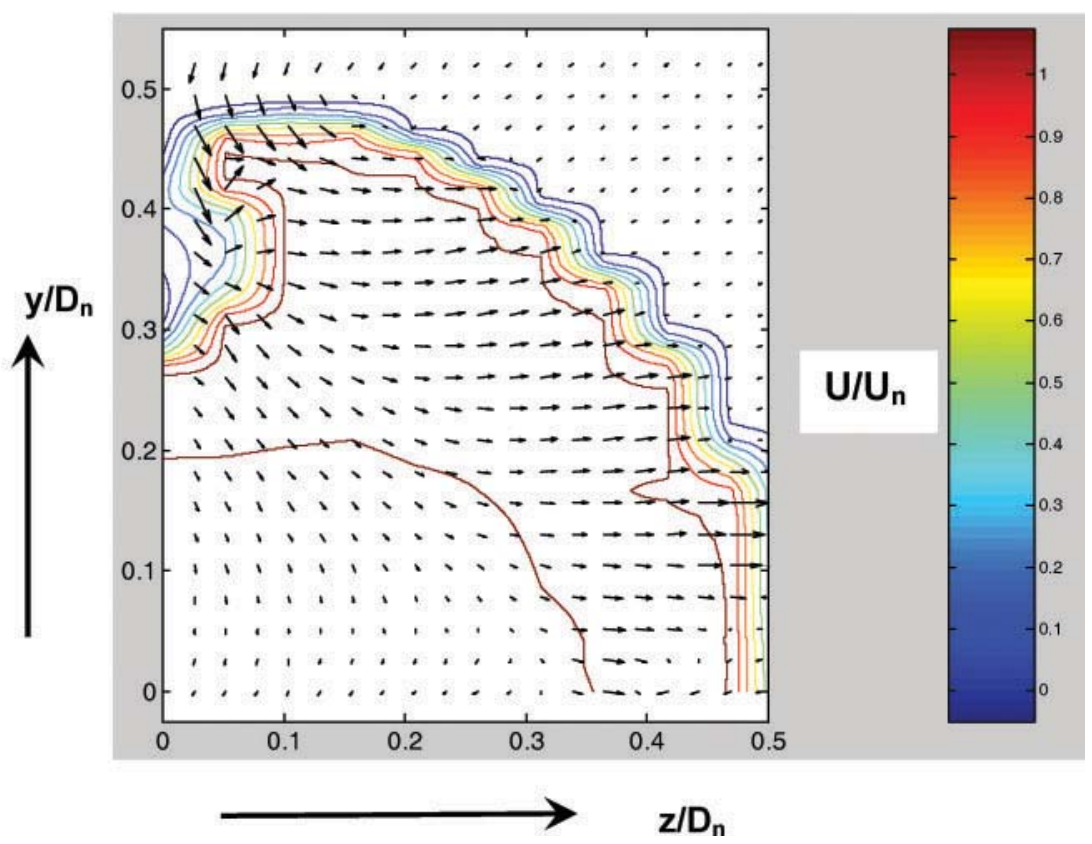

Fig. 2 Jet cross-section distortion (left) [6] and secondary velocity field (right) [8] induced by tabs

speed conditions. Subsequent work to examine the effect of control jet pulsing has been reported in reference [14].

For the present application it is enhanced jet mixing with minimum thrust loss that is the focus of attention and none of the above references using unsteady control jets has properly addressed this regime. Thus, the prime driver for the work reported here was to continue the research work of Behrouzi and McGuirk on steady fluid tabs in reference [13] and their initial studies of pulsed tabs in reference [14] to investigate and compare solid tab, fluid tab, and pulsed fluid tabs, with particular emphasis on the relative performance of these techniques for enhanced jet mixing. The focus of the work is thus shifted in the direction of an active control technique. The control jets add energy to the primary flow in order to influence its mixing with the ambient fluid. In the present paper, only open-loop control is considered. Control parameters (fluid tab jet velocity amplitude and phase) were, at this stage, varied independently of any sensing and feedback of their effect on the main jet behaviour. It is very likely that this form of open-loop control is the technique that may first find engineering application. Any form of closed-loop control would require some sensor signal to drive the feedback loop. The sensor would have to detect the effect of the applied control on the phenomenon being controlled, e.g. the increase in jet spreading or jet entrainment. Since these latter parameters are associated with the first 10 or so diameters of the jet plume, i.e. downstream of the nozzle exit, such sensing would have to be done remotely. It is difficult to see how this could be achieved easily, and there have currently been no published attempts to do this. Hence, it seems sensible to demonstrate first the effectiveness of open-loop control, which is the focus of the present paper.

\section{EXPERIMENTAL TECHNIQUES}

\subsection{Water tunnel facility (WTF) and instrumentation}

Initial experiments were carried out in a water tunnel (for details see Behrouzi and McGuirk [6]). Tab-induced flow behaviour is not strongly influenced by compressibility [6], and water flow studies were considered justified because of the ease (no seeding problems) and cost effectiveness (low-power laser) for measurements of the velocity field using the non-intrusive lased Doppler anemometry (LDA) technique. The LDA instrument measures the velocity of small $(20 \mu \mathrm{m})$ neutrally buoyant seeding particles added to the flow, since these may be assumed to follow the turbulent velocity fluctuations with high fidelity. A view of the test section with a 'clean' (no tabs) nozzle installed and the LDA system in operation is shown in Fig. 3. The axisymmetric 


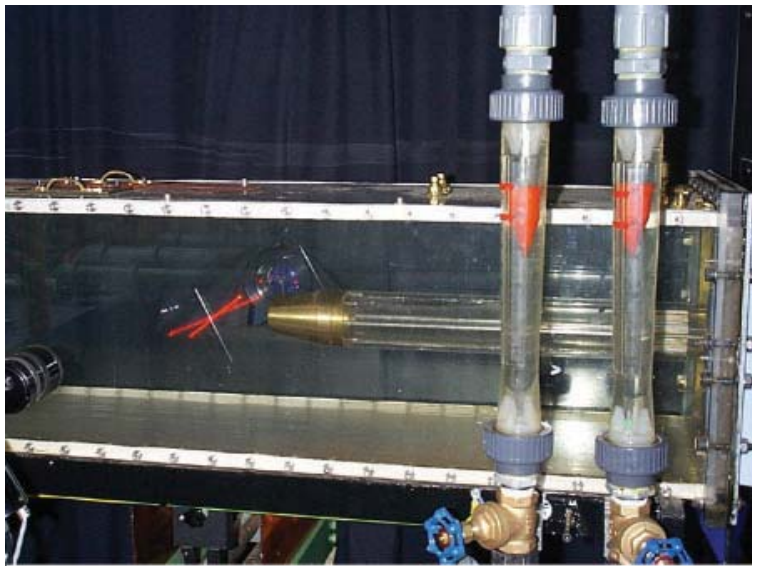

Fig. 3 Water tunnel

nozzle exit diameter and external/internal convergence angles were chosen to form a representative model of a typical aeroengine exhaust nozzle and are shown in Fig. 4. A pair of solid tabs was attached at 12 and 6 o'clock positions in the nozzle exit plane (on the vertical $y$ axis). The tab design was the datum geometry used by Behrouzi and McGuirk [6].

To introduce fluid tabs, two orifices were drilled at the nozzle lip at the same locations as the solid tabs. These were fed via small tubes recessed into the nozzle wall and supplied via an independent pump circuit (Fig. 4 shows just the upper fluid tab supply tube). The mass flow through the fluid tabs could be adjusted to a selected percentage of the primary (core) jet flow. The exit orifice of the fluid tabs was $2 \mathrm{~mm}$ in diameter. A one-component LDA system with a continuous wave (CW) helium-neon laser operating at a power of $15 \mathrm{~mW}$ was used in forwardscatter mode. With the laser beam optical axis oriented as indicated in Fig. 3, the axial ( $x$ component) of velocity was measured. A Bragg cell was used to introduce frequency shifting to provide sensitivity to the flow direction and high turbulence intensity. To minimize statistical error, 40000 samples were gathered during a sampling time of $20 \mathrm{~s}$ from which time-averaged velocities were calculated.

Provision was made to allow pulsation of the control jets. A simple pulse generator unit was fabricated [14] consisting of a DC motor and control unit, a rotating disc, a tachometer, and a rotameter to monitor the disc rotation rate and total fluid tab flowrate respectively. A sketch of the pulse generator unit is given in Fig. 5. The rotating disc was located between end plates; single short inlet/exit pipes fed water to the unit from the pump, and distributed the water to the two fluid tab supply tubes in the nozzle. The inlet/exit pipes were of the same diameter as 12 holes drilled in the rotating disc. With rotation, the holes in the central disc moved past the ends of the inlet/exit pipes in the end plates so that the open area for fluid tab flow increased and decreased with time. The effective time between flow pulses was fixed by the number of holes in the central disc and the disc rotation rate. Pulse rates up to around $12 \mathrm{~Hz}$ were achievable. Expressed as a non-dimensional pulsation frequency (or Strouhal number, $S t=f D_{\mathrm{n}} /$ $\left.U_{\mathrm{n}}\right)$, using the nozzle diameter $\left(D_{\mathrm{n}}\right)$ and bulk exit velocity $\left(U_{\mathrm{n}}\right)$ to define an appropriate flow time scale, $S t$ values in the range 0.0 to 0.45 could be achieved with this system. This range was chosen since previous authors (e.g. Parekh et al. [3]) have documented that primary jets can be excited effectively in this range. A single exit tube was used to feed both fluid tabs equally and simultaneously for what is referred to here as in-phase pulsation. For what is categorized here as $180^{\circ}$ out-of-phase pulsation, upper and lower fluid tabs were fed alternately by using two exit tubes spaced by onehalf of the pitch separation of the holes drilled in the rotating disc.

A maximum fluid tab mass flow ratio (MFR) of around 1 per cent of the core jet primary flow was

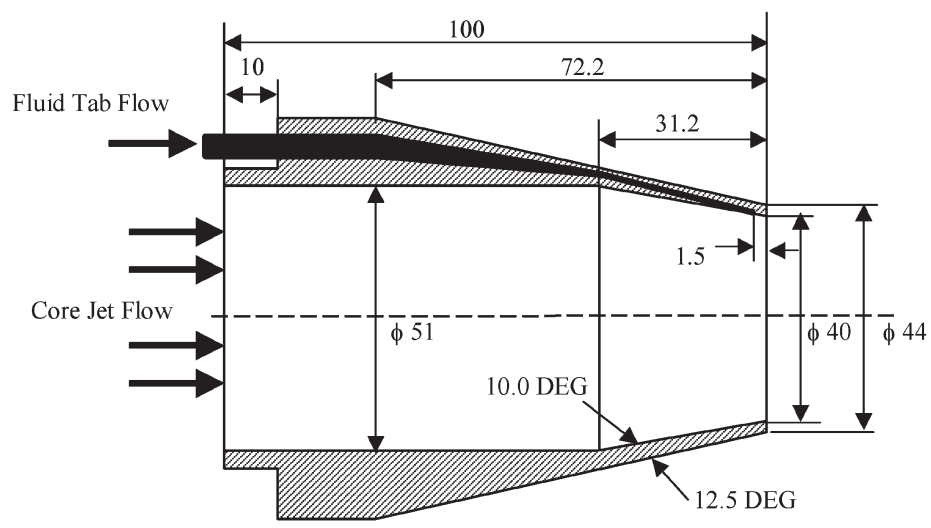

Fig. 4 Nozzle showing one fluid tab passage 


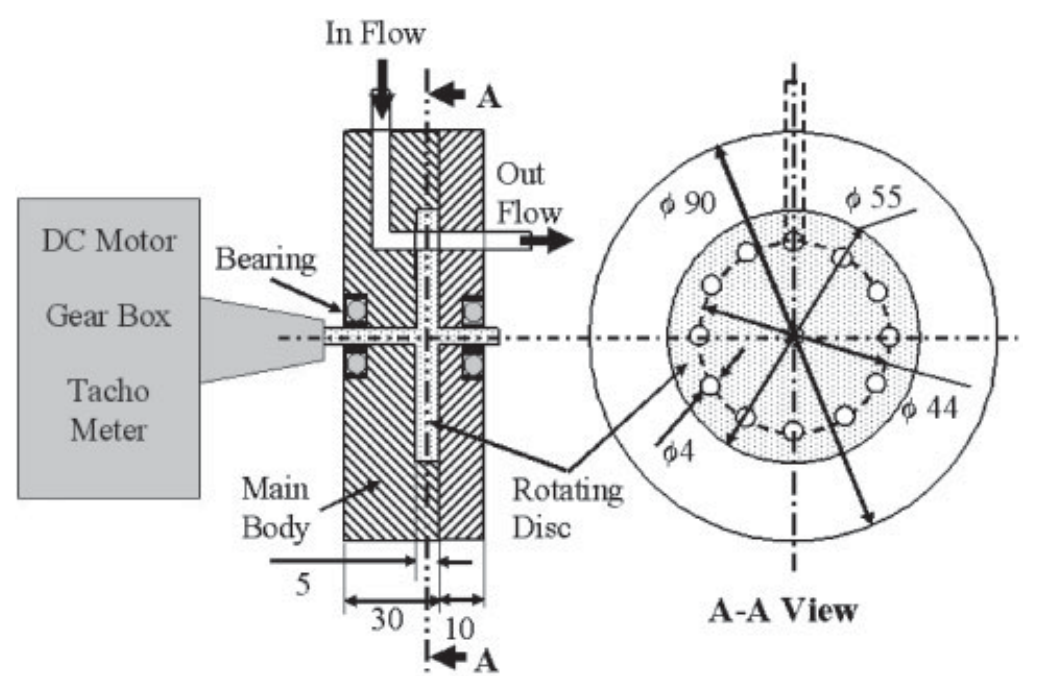

Fig. 5 Rotating disc pulse generator unit

possible with this system. Note that in quantifying the control jet flow, two different approaches have been taken in the literature. For applications relevant to control (delay) of separation from aerofoils, the momentum coefficient, $C_{\mu}$, is often used. This represents the ratio of the momentum flowrate of a single control jet to the primary stream momentum flowrate. From the viewpoint of aeroengine exhaust jet mixing control, where the control jet flow has to be bled from the engine (and is therefore viewed as contributing to a reduction in engine efficiency) and the interest is in minimizing the bleed flow amount, it is the total control jet mass flow ratio, MFR, which is more commonly used to judge the acceptability of the device. Typically if the technology were to require MFR greater than 1 per cent, it would be unlikely to find an application. This is the reason a maximum MFR of $\sim 1$ per cent has been targeted in the tests reported here. Also, MFR and $C_{\mu}$ are simply related (see Notation in the Appendix), so the values of MFR quoted here can easily be converted to $C_{\mu}$ values.

\subsection{High-pressure nozzle test facility (HPNTF) and instrumentation}

A second set of experiments was carried out using a high-pressure nozzle test facility (HPNTF) specially designed for supersonic nozzle flow studies (for details see references $[\mathbf{8}]$ and [13]). An air supply control valve is automatically adjusted to hold the nozzle pressure ratio (NPR) of the nozzle under test to a constant value (typically between 1.5 and 4) to an accuracy of \pm 1 per cent during blow-down testing. Typical blow-down times are between 15 and 30 min depending on NPR value. When the convergent nozzle is supplied with air at a higher than critical NPR $\left(\mathrm{NPR}_{\text {crit }}=1.89\right.$ for ambient temperature air), an underexpanded supersonic jet is formed. To capture jet flowfield development, Schlieren visualization and Pitot pressure measurements were performed using a specially designed Pitot probe. A photograph of a nozzle supplied with twin fluid tabs showing the probe and associated traverse system is given in Fig. 6 [14]. For tests in the HPNTF a convergent nozzle of exit diameter $60 \mathrm{~mm}$ was used, but otherwise the geometry was as in Fig. 5 scaled to this exit nozzle size. For fluid tab studies under high-speed flow conditions two $2 \mathrm{~mm}$ diameter orifices were positioned in the same diametrically opposed configuration/location as the solid tabs (Fig. 6). The fluid tabs were fed from a separate air supply, and a pressure regulator and mass flowrate sensor were included in the fluid tab supply line to monitor the flowrate. Only steady fluid tab flow tests are reported below.

\section{RESULTS}

\subsection{Incompressible flow (water tunnel facility, WTF) measurements}

Measurements were performed along the jet centreline $(x)$ and along orthogonal $y$ and $z$ traverse directions at a distance of five nozzle diameters downstream of the nozzle exit. Complete jet crosssection mapping is the clearest way to quantify jet distortion and spreading behaviour (as seen in Fig. 2). However, it is observed that, although different processes occur in the $y$ and $z$ directions, 


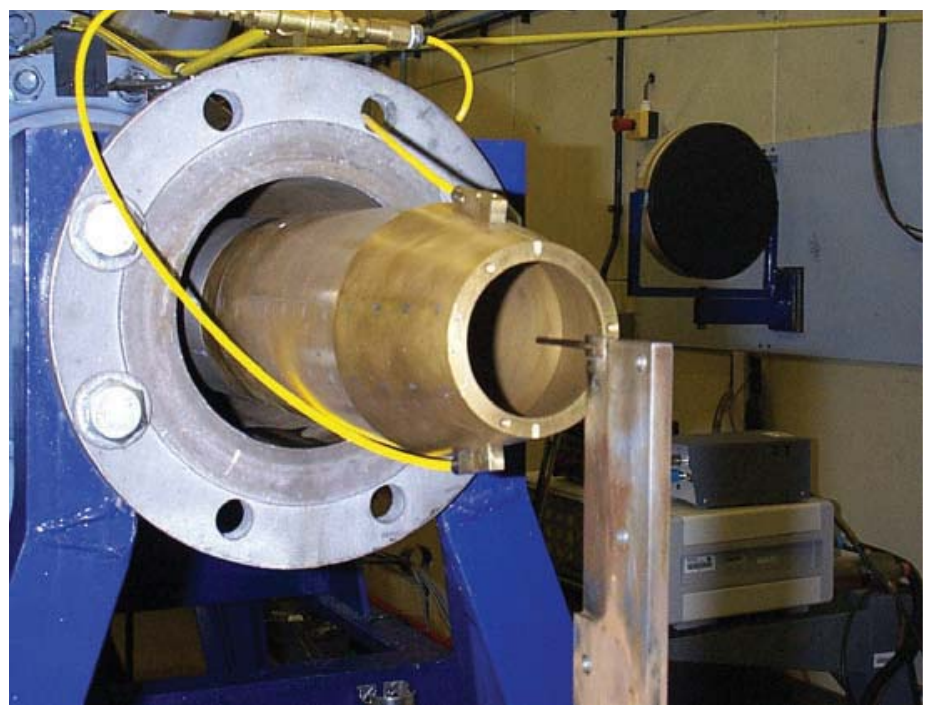

Fig. 6 Nozzle with two fluid tabs in a high-pressure rig, with downstream Pitot probe and traverse

as discussed in section 1, these are in fact closely related. Hence, when a comparative study of different mixing enhancement devices is of interest, as here, examination of profile changes in either the $y$ or $z$ direction is sufficient to assess and rank effectiveness, and a similar observation may be made for axial flow development. Accordingly, in the results shown below, a full set of axial and both $y$ and $z$ profiles are presented for the first case only and subsequently either axial or $z$ profiles are used to compare device performance.

Four sets of tests were performed:

(a) clean nozzle: reference for a natural jet mixing rate;

(b) a pair of solid tabs: reference for an enhanced jet mixing rate;

(c) a pair of steady fluid tabs: effect of varying the fluid tab flowrate;

(d) a pair of pulsed fluid tabs: effect of varying the pulse rate and phase.

The emphasis is placed on the centre-line profile and on transverse profiles at a given downstream distance, since these allow clear cross-comparison between the different configurations of the two important parameters for jet mixing mentioned above, i.e. potential core length and jet decay rate.

\subsubsection{Effect of the fluid tab flowrate (steady flow)}

LDA measurements were performed for two tab mass flow ratios, MFR $=0.5$ and 1 per cent. The velocity ratio between the radial inward velocity of the control jets and the core jet axial velocity $\left(V_{\mathrm{j}} / U_{\mathrm{n}}\right)$ was 1.0 and 2.0 for these two MFR cases. Figures 7 and 8 show the measured mean axial velocity along the jet centre-line and along both $y$ and $z$ traverse lines at $x / D_{\mathrm{n}}=5$ for the clean nozzle as well as for the solid tab and fluid tab cases. The clean nozzle results show that the potential core length is $\sim 4.5$ nozzle diameters (defined as the axial location where the centre-line velocity begins to decrease from its initial value). The effect of introducing solid tabs is to reduce the potential core length significantly, to around $x / D_{\mathrm{n}}=1.5$. The decay rate (i.e. the absolute magnitude of the axial velocity gradient $\mathrm{d} U / \mathrm{d} x$ ) is also enhanced in the region between 1.5 and $\sim 8 x /$ $D_{\mathrm{n}}$. Both of these effects are near-field effects, since the jet decay rate returns to its clean nozzle value after $x / D_{\mathrm{n}} \sim 8$.

The $z$ profiles for the clean nozzle show a jet halfwidth of $\sim 0.6 D_{\mathrm{n}}$ at $x / D_{\mathrm{n}}=5$. Solid tabs increase the half-width in this plane substantially; the profile also shows off-centre-line peaks, indicating jet crosssection distortion due to the strong streamwise vortex created. The fluid tab results show that the $\mathrm{MFR}=0.5$ per cent case gave only a small improvement in jet mixing compared to the natural jet. The $\mathrm{MFR}=1$ per cent fluid tab, however, approached the solid tab results. The potential core length for the $\mathrm{MFR}=1$ per cent fluid tab was also reduced to $x /$ $D_{\mathrm{n}}=1.5$, although the enhanced rate of decay was only sustained up to around $x / D_{\mathrm{n}} \sim 3$. On the other hand, the increased jet width in the $z$ direction profiles and the tendency towards bifurcation of the profile at $x / D_{\mathrm{n}}=5$ (see Fig. 8) were very similar 


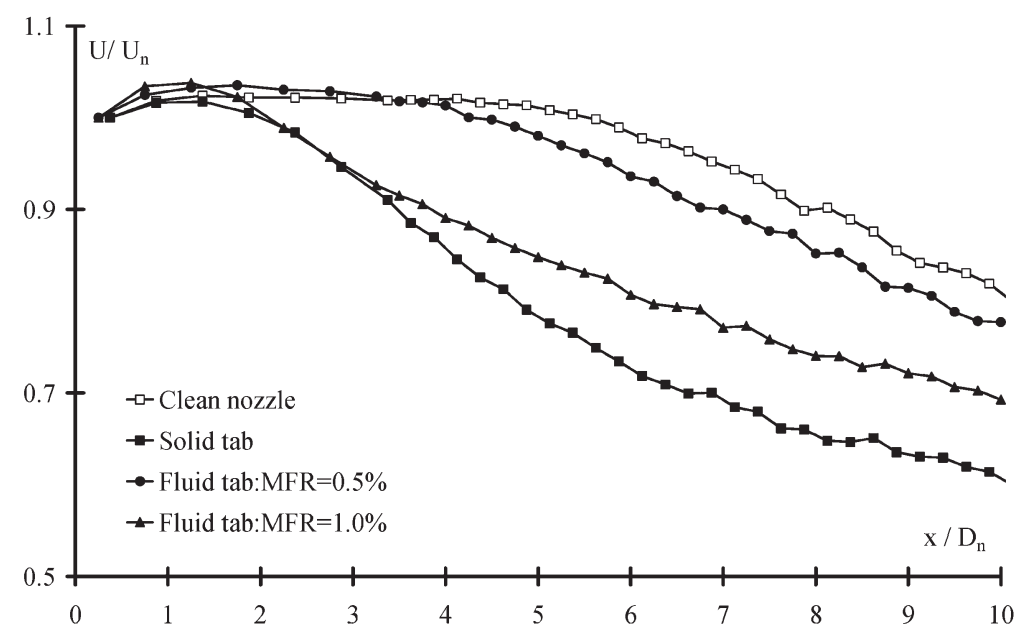

Fig. 7 Centre-line velocity for two tab flowrates

between the MFR = 1 per cent fluid tab and the solid tab data. The $y$ direction profiles show good symmetry of the flow about both transverse axes for the clean jet case. The three-dimensional nature of the jet development with tabs is indicated by the $y$ and $z$ profiles individually being symmetric about one axis, but the shapes in each direction are quite different. The jet width is reduced in the $y$ direction relative to the clean case due to the strong inwards motion created by the tabs, as illustrated in Fig. 1. The strongest inwards motion is observed for the solid tabs. The MFR $=0.5$ per cent case shows some movement away from the clean jet case, but only marginally. The MFR $=1$ per cent fluid tab inwards deflection is very similar to the solid tab result. Note that in this direction no bifurcation is observed and all profiles are roughly Gaussian in shape. Note also that the effectiveness of the various mixing enhancement devices is the same whether the $y$ or $z$ direction profiles are examined. Hence for other cases presented below only axial or $z$ direction data are used.
The important evidence provided by these data is that, for incompressible jets, a steady fluid tab nozzle is clearly capable of producing a similar effect on jet mixing as achieved by the use of solid tabs.

\subsubsection{Effect of fluid tab pulse frequency}

Excellent performance in the steady flow fluid tab experiments was achieved for $\mathrm{MFR}=1$ per cent, whereas little increased mixing had been observed for $\mathrm{MFR}=0.5$ per cent. For measurements with pulsed fluid tabs, however, it was known from earlier studies (e.g. reference [12]) that the introduction of unsteadiness into the control jets would enhance their effectiveness. Hence for pulsed jet testing the time-averaged fluid tab flowrate was set at $\overline{\mathrm{MFR}}=0.5$ per cent. The design of the pulsation unit described above clearly indicates that the open area for fluid tab flow will vary with time, implying that the tab flowrate would increase and decrease above its time-averaged value.
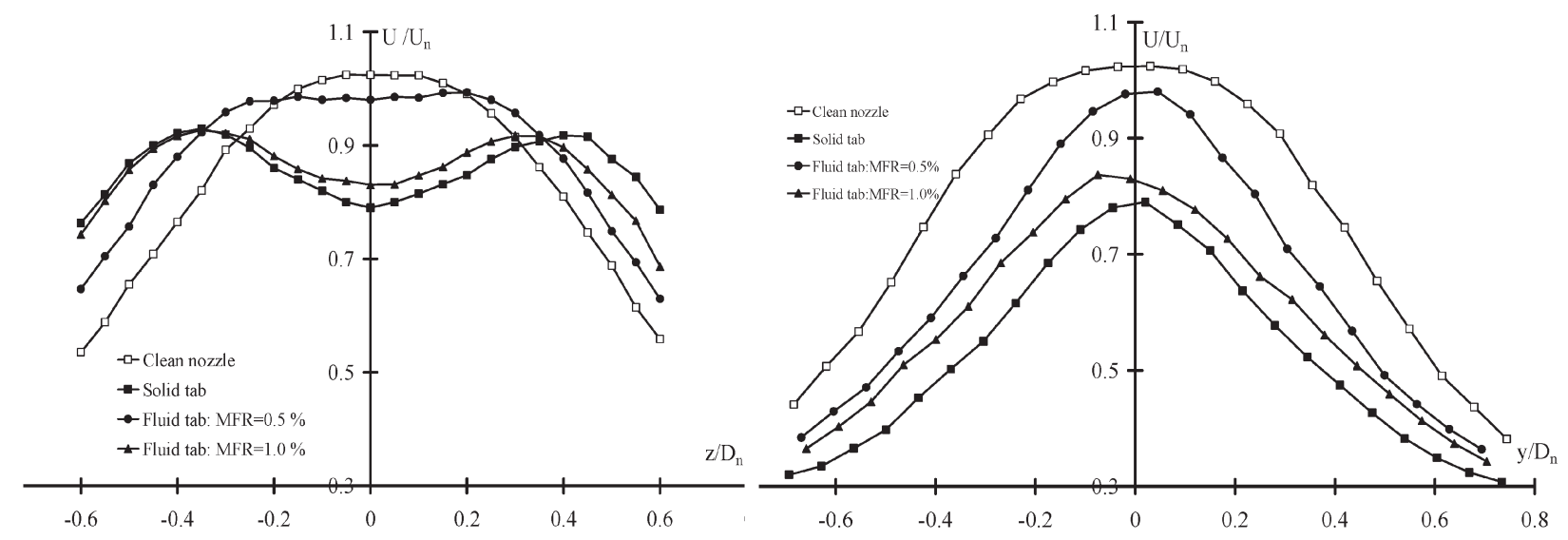

Fig. 8 Transverse axial velocity profiles for two tab flowrates: left $z$ profiles, right $y$ profiles 
Figure 9 presents the effect of varying the fluid tab flow pulse frequency at a time-averaged MFR of 0.5 per cent on the development of axial mean velocity along the jet centre-line. Figure 10 presents measured axial velocity profiles along the $z$ direction at $x / D_{\mathrm{n}}=5$. Tests were performed for two pulse frequencies of 2 and $4 \mathrm{~Hz}$; these values were chosen since they approach the non-dimensional frequency value observed in other works to give maximum effect on the primary jet. Pulsing the tab flow at a fixed time-averaged MFR is seen to be very effective. For example, the core jet velocity decay rate has increased by pulsing the fluid tab flow such that at $10 D_{\mathrm{n}}$ downstream the centre-line velocity has been reduced from the steady tab flow value of $U / U_{\mathrm{n}}=0.8$ to $U / U_{\mathrm{n}}=0.7$ (relative to the solid tab result of $U /$ $U_{\mathrm{n}}=0.62$ ). Jet decay with only 0.5 per cent timeaveraged fluid tab flow but with pulsations at $2 \mathrm{~Hz}$ achieves the same performance as a 1 per cent steady fluid tab flow, showing that pulsations lead to more effective use of control jet mass flow. It is for this reason that the average MFR level was reduced in the present pulsating tests compared to the steady data. Behrouzi and McGuirk [13] identified that, for optimum mixing enhancement, the streamwise vorticity created by the fluid tab jet bending over into the primary jet flow should be located in the primary jet shear layer rather than penetrating too far into the primary jet. Enhancement of the jet mixing rate does not improve when the pulsation frequency is doubled. The Strouhal number of the pulsation $\left(S t=f D_{\mathrm{n}} / U_{\mathrm{n}}\right)$ is 0.08 at $2 \mathrm{~Hz}$ and 0.16 at $4 \mathrm{~Hz}$. The latter is closer to the Strouhal number $(S t \sim 0.2)$ at which most effective mixing and jet spread was observed in the experiments of Parekh et al. [3], but the present data show little effect of Strouhal number. One reason for this is that, whereas the study of Parekh et al. [3] attempted to use pulsations to excite non-linear behaviour of the natural flapping mode instability of the jet shear layer, the present design intent is to induce a strong streamwise vortex into the shear layer vicinity. In the present scenario the velocity ratio between the fluid tab control jet and the core jet is much larger $(\sim 2-3$ times) than the ratio used in reference [3] to excite the instability wave. The basic mechanism for the jet response here is therefore more akin to the jet in crossflow studies, and the optimum Strouhal number of the pulsation frequency may well be different. More work is needed to clarify this.

\subsubsection{Effect of the fluid tab flow pulse phase}

Figure 11 shows the effect of altering the fluid tab pulse phase (at a fixed average MFR of 0.5 per cent and a fixed pulse frequency of $2 \mathrm{~Hz}$ ) on the measured axial mean velocity along the jet centre-line. Tests were performed for two cases, i.e. in-phase and $180^{\circ}$ out-of-phase pulsations (fluid tabs pulsing alternately); all other test conditions were kept fixed. The effect of the pulse phase is substantial. The core jet axial velocity at $x / D_{\mathrm{n}}=10$ was reduced (relative to the clean nozzle measurements as the datum) by 8 and 30 per cent with 0.5 per cent steady fluid tabs and a pair of solid tabs respectively. The reduction was measured to be 17 and 28 per cent with $2 \mathrm{~Hz}$ pulsed fluid tabs for in-phase and $180^{\circ}$ out-of-phase respectively. This indicates that 0.5 per cent $2 \mathrm{~Hz}$

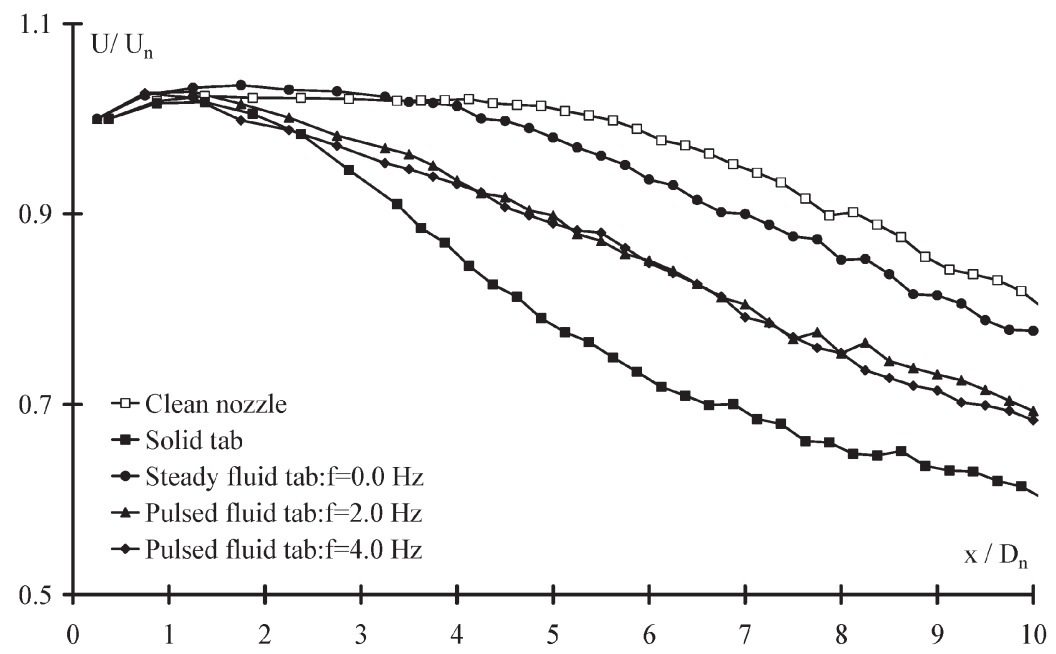

Fig. 9 Centre-line velocity for two pulse frequencies (MFR $=0.5$ per cent) 


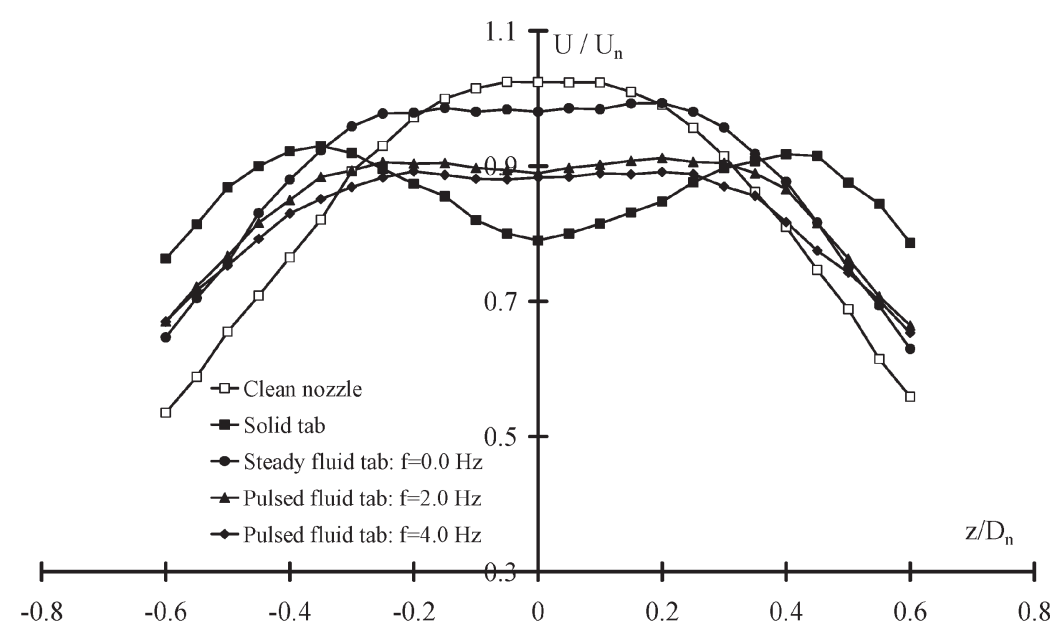

Fig. 10 Transverse $(z)$ axial velocity profiles for two pulse frequencies (MFR $=0.5$ per cent)

out-of-phase pulsed fluid tabs produce essentially the same effect on the core jet as two solid tabs.

\subsection{High-speed compressible (high-pressure nozzle test facility, HPNTF) measurements}

It is clearly of interest to extend the low-speed investigations of fluid tabs conducted in the WTF to high-speed compressible flow. These measurements were carried out in the HPNTF rig described fully in references [8] and [14]. The comparative tests covered a similar series of increasing flow complexity: clean nozzle, solid tabs, and fluid tabs. In these highpressure tests so far only steady fluid tabs have been studied; future work is planned to extend the experiments to pulsed flow. Tests were carried out at nozzle pressure ratios of $\mathrm{NPR}=1.5$ and 3 . Flow visualization was initially performed for a range of NPR values. Figure 12 shows Schlieren pictures taken of a clean and a solid tabbed nozzle at NPR $=2.32$. Under high Mach number compressible flow conditions, additional phenomena appear in the form of an underexpanded jet. A series of expansion and compression waves appear as illustrated in the Schlieren pictures. These become weaker with distance as the jet expands back to atmosphere pressure. The shock cells extend to the end of the primary jet potential core at $\sim 4 D_{\mathrm{n}}$ (only the first five shock cells are visible in the image shown). With introduction of two solid tabs, extra shock and expansion waves appear. In the plane containing the tabs $(x-y)$, Fig. 12 shows that the inwards flow in this plane associated with the streamwise vortices (described above and illustrated in Fig. 1) causes two effects: a reduction in jet

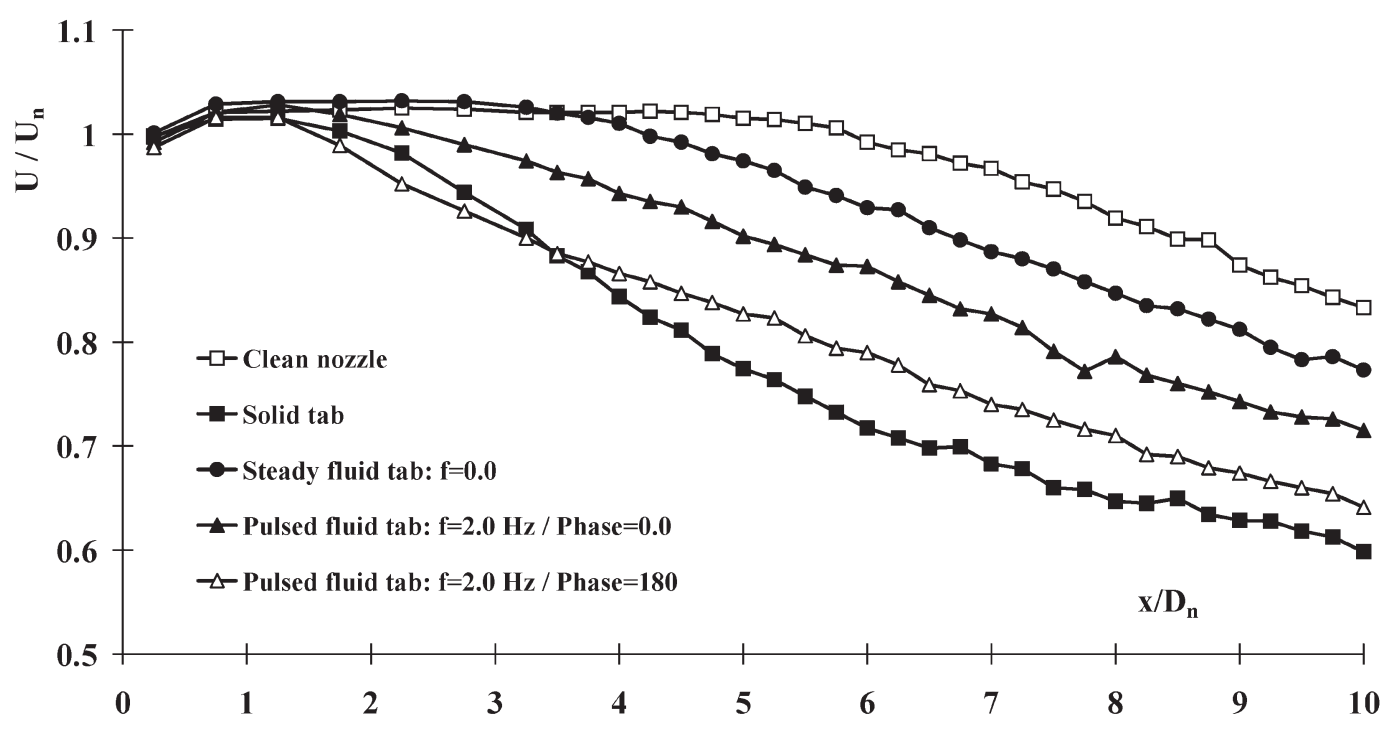

Fig. 11 Centre-line axial velocity at $M F R=0.5$ per cent and pulse frequency of $2 \mathrm{~Hz}$, in-phase and out-of-phase 

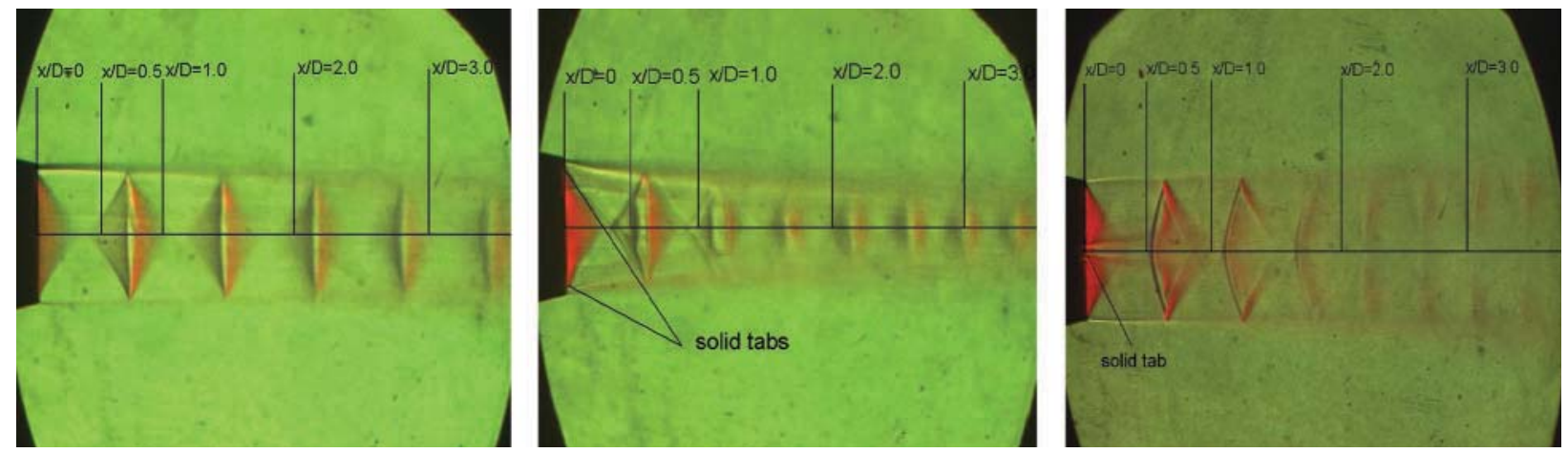

Fig. 12 Schlieren visualization for NPR = 2.32: left, clean jet; centre, with tabs, $x-y$ plane; right, with tabs, $x-z$ plane

diameter as the plume develops and a more rapid reduction in shock cell strength with downstream distance compared to the clean nozzle case. This latter effect is associated with the enhanced spreading of the jet shear layer and the associated weaker reflection of oblique shock and compression waves. In the orthogonal $x-z$ plane, Fig. 12 demonstrates the vortex-induced outwards movement and the strongly curved nature of the shockwaves caused by threedimensional flow effects. Similar features are observed for steady fluid tabs.

\subsubsection{Effect of the fluid tab flowrate (steady flow)}

The decay of Pitot pressure along the nozzle centreline was measured for clean, solid tab, and fluid tab configurations for various MFR values and two NPRs. Figures 13 and 14 provide results for $\mathrm{NPR}=1.5$ and 3.0 respectively. The effect of the fluid tab flowrate is very significant. At NPR $=1.5$, even for the lowest tab flowrate of MFR $=0.47$ per cent the fluid tab performance is marginally better than the solid tab case. At MFR $=0.67$ per cent the fluid tab performance far exceeds the solid tab data. The core jet potential core length has decreased even more and the centre-line decay rate increased, although, as already noted, the benefits of all rapid mixing devices cease after the initial region $(x)$ $D_{\mathrm{n}}>8$ ). A further increase to MFR $=0.93$ per cent shows only a marginal improvement, so an optimum fluid tab flowrate clearly exists. Optimum in the present context means the efficient use of the minimum control jet flowrate (to minimize engine bleed), for the maximum effect on the primary jet, e.g. the maximum increase in primary jet mass flow with downstream distance. The existence of an optimum is undoubtedly associated with the variation of control jet penetration with MFR. To achieve

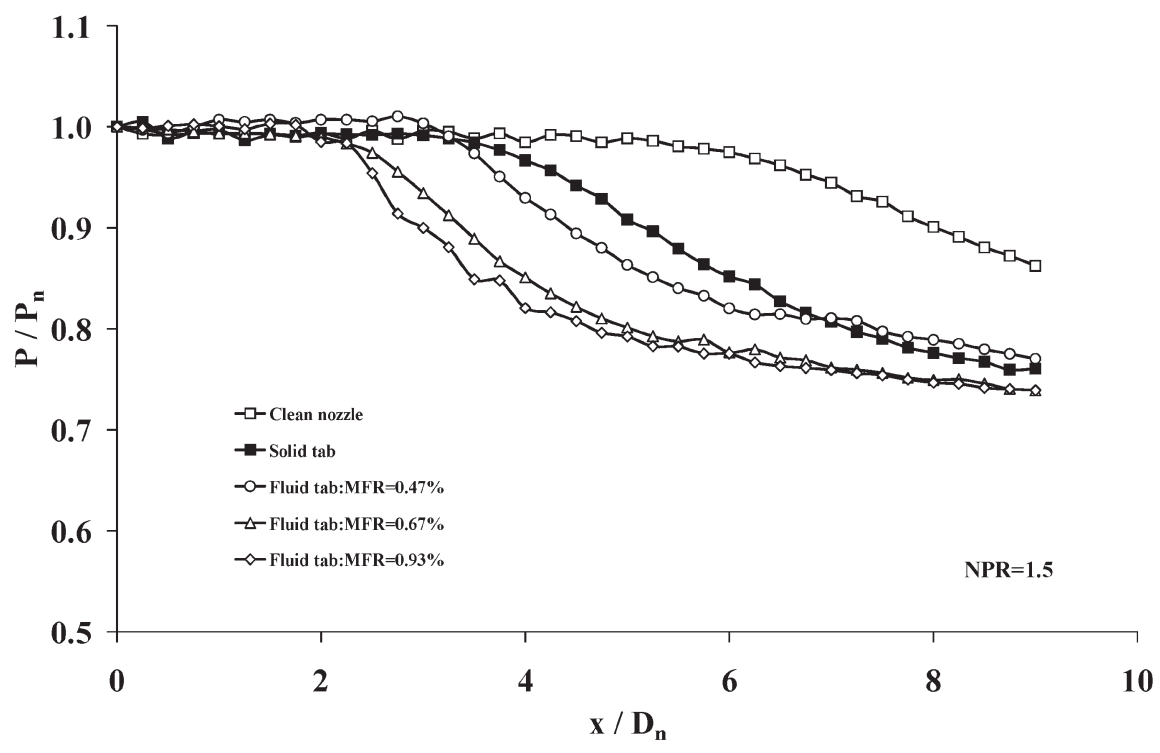

Fig. 13 Axial velocity decay for NPR $=1.5$, various MFRs 


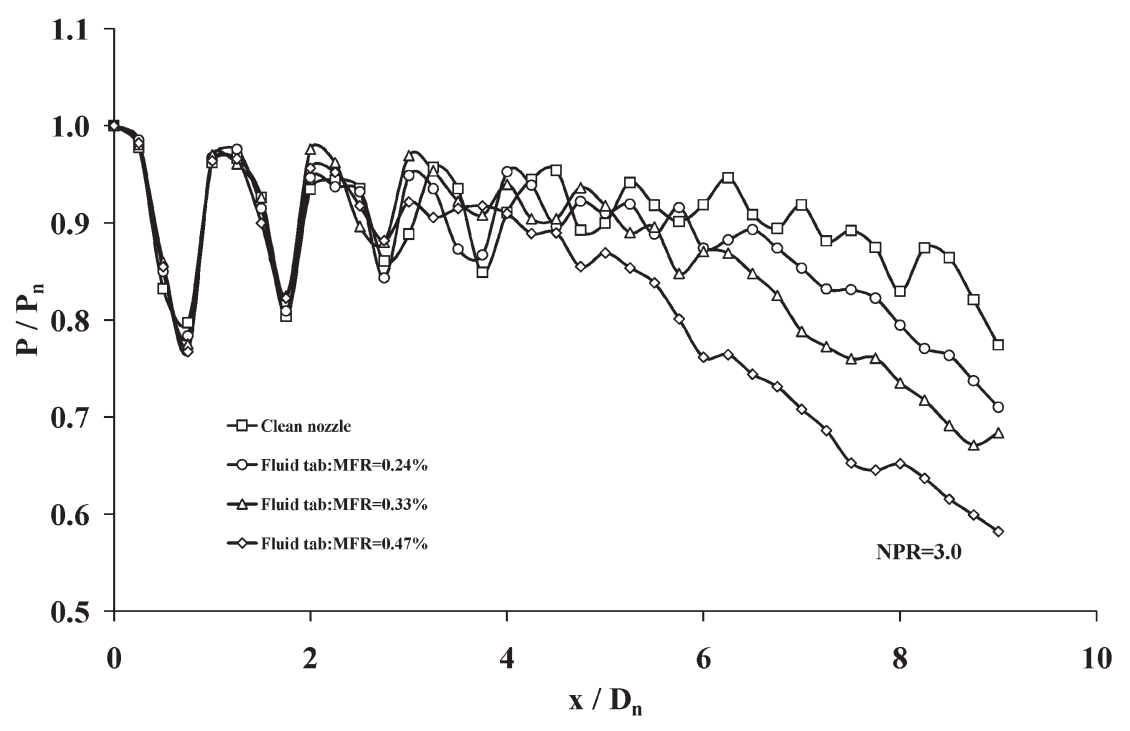

Fig. 14 Axial velocity decay for NPR $=3.0$, various MFRs

strong interaction between the fluid tab-induced streamwise vortices and the primary jet shear layer, it is important not to increase the MFR too much for a fixed control jet exit orifice size, since this will increase the velocity ratio $\left(V_{\mathrm{j}} / U_{\mathrm{n}}\right)$ and hence will move the control jet trajectory away from the primary jet shear layer growing from the nozzle lip.

The data for NPR $=3.0$ show similar behaviour; in this case MFR levels even lower were used $(0.24$, 0.33 , and 0.52 per cent) and all showed clear evidence of enhanced mixing rates. The fluid tab flowrate required to achieve a given level of enhanced jet mixing seems to be lower in the highspeed data than in the incompressible flow tests. Although similarities exist between low-speed and high-speed tests, differences are clearly present. At $\mathrm{NPR}=1.5$ the jet exit Mach number is around 0.8 and at NPR $=3$ the nozzle is obviously choked and underexpanded, as illustrated in Fig. 13, so the initial shear layer in both cases is clearly compressible. It is known that a compressibility effect exists in shear layers at sufficiently high Mach numbers [15], which reduces the natural spreading rate of the layer. It is possible that the effect of the streamwise vortices induced by the fluid tab control jets on the shear layer interact in a subtle way with the effects of compressibility on the shear layer. This clearly cannot be present in the low-speed tests. More detailed measurements of the high Mach number round the jet shear layer and the streamwise vortex in the near nozzle exit region are needed to understand this, and such studies are already underway and reported in Feng and McGuirk [8, 16, 17], but these initial results of flow control of high-speed jets with fluid tabs are highly promising.

\section{CONCLUSIONS}

Evidence has been provided in this paper that fluid tabs create similar streamwise vorticity-induced enhanced mixing processes as solid tabs. The control jet flowrate demand to achieve these effects seems reasonable from an engine bleed viewpoint. Potential core length reductions, enhanced entrainment and spreading, and increased turbulence levels are all observed. Fluid tabs, as noted above, provide the extra flexibility that they can be switched off when not required, but they also offer the possibility of velocity ratio manipulation for varying core jet conditions. Indeed, the possibility of using control jet pulsation frequency and phase as active control parameters is also present and was studied in the present measurements. The mixing effectiveness achieved was shown to be optimized by a suitable choice of pulsation frequency and phase. Extension of the results observed at low flow speeds into the compressible flow range at which aeroengine propulsion nozzle jets operate was also studied. In general, similar effects were observed, although to date pulsed jets have not been studied. An additional effect was seen, the cause of which was possibly the interaction between compressibility effects and vorticity-enhanced spreading effects. Further data are needed to confirm this, but it is believed the use of pulsed fluid tabs for active control of primary jet plume mixing is certainly worthy of further study. 


\section{REFERENCES}

1 Seiner, J. M., Dash, S. M., and Kenzakowski, D. C. Historical survey on enhanced mixing in scramjet engines. Am. Inst. Aeronaut. Astronaut. J. of Propulsion and Power, 2001, 17, 1273-1286.

2 Rockwell, D. O. External excitation of planar jets. Trans. ASME, J. Appl. Mechanics, 1972, 39, 883-890.

3 Parekh, D. E., Kibens, V., Glezer, A., and Wiltse, J. M. Innovative jet flow control: mixing enhancement experiments. AIAA-96-0308, 1996.

4 Bradbury, L. J. S. and Khadem, A. H. The distortion of a jet by tabs. J. Fluid Mechanics, 1975, 70, 801-813.

5 Samimy, M., Zaman, K. B. M. Q., and Reeder, M. F. Effect of tabs on the flow and noise field of an axisymmetric jet. Am. Inst. Aeronaut. Astronaut. J., 1993, 31, 609-615.

6 Behrouzi, P. and McGuirk, J. J. Experimental studies of tab geometry effects on mixing enhancement of an axisymmetric jet. JSME Int. J., Ser. B, 1998, 41, 908-917.

7 Bohl, D. G. and Foss, J. F. Near exit plane effects caused by primary and primary-plus-secondary tabs. Am. Inst. Aeronaut. Astronaut. J., 1999, 37, 192-201.

8 Feng, T. and McGuirk, J. J. LDA measurements of underexpanded jet flow from an axisymmetric nozzle with solid tabs. In 3rd AIAA Flow Control Conference, San Francisco, California, June 2006, paper AIAA-2006-3702.

9 AGARD Conference Proceedings 534 on Computational and experimental assessment of jets in crossflow. AGARD-CP-534, November 1993.

10 Margason, R. J. Fifty years of jet in crossflow research. AGARD 543, 1993, pp. 1-41.

11 Bray, J. P. and Garry, K. P. Optimisation of air jet vortex generators with respect to system design parameters. Aeronaut. J., 1999, 103, 475-480.

12 Bons, J. P., Sondergaard, R., and Rivir, R. B. Turbine separation control using pulsed vortex generator jets. Trans. ASME, J. Turbomachinery, 2001, 55, 198-206.

13 Behrouzi, P. and McGuirk, J. J. Jet mixing enhancement using fluid tabs. In 2nd AIAA Flow Control Conference, Portland, Oregon, 28 June-1 July 2004.

14 Behrouzi, P. and McGuirk, J. J. Flow control of jet mixing using a pulsed fluid tab nozzle. In 3rd AIAA Flow Control Conference, San Francisco, California, June 2006.

15 Papamoschou, D. and Roshko, A. The compressible turbulent shear layer: an experimental study. J. Fluid Mechanics, 1988, 197, 453-477.

16 Feng, T. and McGuirk, J. J. LDA measurements in high Mach number axisymmetric jet shear layers. In 5th Turbulent Shear Flow Phenomena Symposium, Munich, Germany, August 2007.

17 Feng, T. and McGuirk, J. J. LDA measurements of heated and unheated underexpanded jets from axisymmetric nozzles. In International Symposium on Air breathing engines, Beijing, China, September 2007.

\section{APPENDIX}

\section{Notation}

$a$

$C_{\mu}$

$D_{\mathrm{j}}$

$D_{\mathrm{n}}$

$f$

$\dot{m}_{\mathrm{j}}$

$\dot{m}_{\mathrm{n}}$

$M$

MFR

$N_{\mathrm{j}}$

NPR

$p$

$p_{\text {atm }}$

$P$

$P_{\mathrm{n}}$

St

$U$

$U_{\mathrm{n}}$

$V_{\mathrm{j}}$

$x$

$y$

$z$ speed of sound

momentum coefficient: (single) control jet momentum flowrate/primary jet momentum flowrate, $\left(\mathrm{MFR} / N_{\mathrm{j}}\right)$ $\left(V_{\mathrm{j}} / U_{\mathrm{n}}\right)$

control jet (fluid tab) exit orifice diameter $(\mathrm{m})$

primary jet nozzle diameter (m) frequency of control jet pulsation (Hz) control jet (fluid tab) mass flowrate (total), $N_{\mathrm{j}} \rho_{\mathrm{j}} V_{\mathrm{j}} \pi D_{\mathrm{j}}^{2} / 4(\mathrm{~kg} / \mathrm{s})$

primary jet mass flowrate, $\rho_{\mathrm{n}} U_{\mathrm{n}}$ $\pi D_{\mathrm{n}}^{2} / 4(\mathrm{~kg} / \mathrm{s})$

Mach number of primary nozzle flow $\left(M=U_{\mathrm{n}} / a\right)$

mass flow ratio: mass flowrate in control jets (total)/primary jet mass flowrate, $\dot{m}_{\mathrm{j}} / \dot{m}_{\mathrm{n}}$ number of control jets (fluid tabs) primary jet nozzle pressure ratio, $P_{\mathrm{n}} /$ $p_{\text {atm }}$ static pressure $(\mathrm{Pa})$ ambient atmospheric pressure $(\mathrm{Pa})$ total pressure $(\mathrm{Pa})$ primary nozzle supply total pressure (Pa)

Strouhal number, non-dimensional pulsation frequency $\left(S t=f D_{\mathrm{n}} / U_{\mathrm{n}}\right)$ axial velocity (time-averaged) ( $x$ direction) $(\mathrm{m} / \mathrm{s})$

primary jet bulk velocity, $\dot{m}_{\mathrm{n}} /\left(\rho_{\mathrm{n}} \pi D_{\mathrm{n}}^{2} / 4\right)(\mathrm{m} / \mathrm{s})$

radial velocity (time-averaged) ( $r$ direction) $(\mathrm{m} / \mathrm{s})$

control jet bulk velocity $\dot{m}_{\mathrm{j}} /\left[N_{\mathrm{j}}\left(\rho_{\mathrm{j}} \pi D_{\mathrm{j}}^{2} / 4\right)\right](\mathrm{m} / \mathrm{s})$ axial direction (primary nozzle discharge axis) (m) transverse (radial) direction (axis connecting tabs or control jets) (m) transverse (radial) direction (orthogonal to $y$ ) (m) 